\title{
Breast cancer risk associated with genotypic polymorphism of the genes involved in the estrogen-receptor-signaling pathway: a multigenic study on cancer susceptibility
}

\author{
Jyh-Cherng $\mathrm{Yu}^{1}$, Huan-Ming $\mathrm{Hsu}^{1,2}$, Shou-Tung $\mathrm{Chen}^{3}$, Giu-Cheng Hsu ${ }^{4}$, \\ Chiun-Sheng Huang ${ }^{5}$, Ming-Feng Hou ${ }^{6}$, Yi-Ping $\mathrm{Fu}^{2}$, Ting-Chih Cheng ${ }^{2}$, Pei-Ei $\mathrm{Wu}^{2}$ \& \\ Chen-Yang Shen ${ }^{2,7, *}$ \\ ${ }^{1}$ Departments of Surgery, Tri-Service General Hospital, Taipei, Taiwan; ${ }^{2}$ Institute of Biomedical Sciences, \\ Academia Sinica, Taipei, Taiwan; ${ }^{3}$ Division of General Surgery, Department of Surgery, Changhua Christian \\ Hospital, Changhua, Taiwan; ${ }^{4}$ Department of Radiology, Tri-Service General Hospital, Taipei, Taiwan; \\ ${ }^{5}$ Department of Surgery, National Taiwan University Hospital, Taipei, Taiwan; ${ }^{6}$ Department of Surgery, \\ Kaohsiung Medical University Hospital, Kaohsiung, Taiwan; ${ }^{7}$ Life Science Library, Academia Sinica, Taipei, \\ Taiwan
}

Received 5 September 2005; accepted 2 January 2006

(c) 2006 National Science Council, Taipei

Key words: breast cancer, E-cadherin, estrogen, estrogen receptor, genetic polymorphism, MTA1, MTA3, snail

\section{Summary}

The reproductive hormone, estrogen, contributes to the development of breast cancer by binding to the estrogen receptor (ER) in the nucleus, triggering cell growth and tumor promotion. In addition to its role in regulating target genes and signaling pathways involved in cell cycle progression, the ER-signaling pathway may regulate the expression of chromatin-remodeling gene, Metastasis-associated 3 (MTA3), or interact with chromatin-remodeling protein, Metastasis-associated 1 (MTA1). The invasion-suppressor gene, $E$-Cadherin $(E$-Cad), has recently been identified as a downstream target gene regulated by the ER-MTA3 pathway via the transcriptional repressor, Snail, and the ER-MTA3-Snail-E-Cad pathway has therefore been evoked to explain the clinical observation that ER expression in breast cancer is generally associated with a better clinical outcome. Since E-Cad may play an initiating role during breast tumorigenesis, we hypothesized that this ER-signaling pathway may also determine susceptibility to breast cancer, and examined this in a multigenic case-control study of 468 incident breast cancer patients and 470 healthy controls by genotyping the single nucleotide polymorphisms (SNPs) in five genes (ER, MTA3, Snail, E-Cad, and MTA1) in the ER-signaling pathways. Support for this hypothesis came from the observations that (a) with the exception of Snail, which interacted differently with reproductive risk factors in relation to breast cancer risk, there was a joint effect of the SNPs of these genes and estrogen-related risk factors (age at first full-term pregnancy and obesity, measured by the body mass index) on breast cancer risk $(p<0.05)$; (b) a trend toward increased risk of developing breast cancer was seen in women harboring a greater number of putative high-risk genotypes of these genes in ER-signaling pathways; (c) this association between risk and the number of putative high-risk genotypes was stronger and more significant in women thought to have experienced higher estrogen level, i.e., obese women; and (d) the risk effect conferred by obesity was only significant in women with a higher number of putative high-risk genotypes of the ERsignaling genes. These epidemiological findings highlight the role of newly identified novel ER-related

*To whom correspondence should be addressed. E-mail: bmcys@ibms.sinica.edu.tw 
pathways in breast cancer development and provide a more comprehensive picture of the tumorigenic effect of estrogen in breast cancer development.

\section{Introduction}

The contribution of the reproductive hormone, estrogen, to the development of breast cancer has been well documented in epidemiological and molecular and cell biology studies. Well-established risk factors for breast cancer, including age at menarche, age at menopause, parity, and age at first full-term pregnancy (FFTP), play a role in breast cancer by hormonal mechanisms [1, 2]. Hypotheses in which estrogen is involved in tumorigenesis are based on the general concept that cell division is crucial for cancer development and that reproductive risk factors increase mitotic activity in the breast epithelium, resulting in increased cancer risk [3, 4]. Estrogen triggers cell growth and tumor promotion by binding to the estrogen receptor (ER) in the nucleus, leading directly to the expression of many target genes involved in cell cycle progression and indirectly to the activation of signaling pathways, and resulting in proliferation [5, 6]. Interestingly, the downstream genes regulated by estrogen-ER signaling have recently been expanded to include some not directly involved in cell growth control. Metastasisassociated 3 (MTA3) is one such gene [7, 8], the major function of which is suggested to be the formation of a repressive chromatic structure, which shuts down the expression of target genes [9]. Recently, ER-regulated activation of MTA3 was shown to result in reduced expression of the transcriptional repressor, Snail [8]. Since increased Snail expression has been shown in cell line studies to lead to decreased expression of the cell adhesion molecule, E-Cadherin (E-Cad) [10], thus allowing tumor cells a greater potential to invade and metastasize, the ER-MTA3-Snail-E-Cad pathway has been evoked to explain the clinical observation that ER expression in breast cancer is generally associated with a better clinical outcome [11-13]. However, in addition to tumor progression, there is great interest in defining whether this ER-signaling pathway determines susceptibility to breast cancer and contributes to breast cancer initiation. The rationale underlying this hypothesis is that: (a) not only is ER status considered as a prognostic marker, but genotypic polymorphism of $E R$ is associated with risk of breast cancer development
[14]; (b) in our genome-wide study [15, 16], genetic deletion at $16 \mathrm{q} 21$, a region harboring $E$-Cad, was defined as an initiating step in breast cancer development; and (c) germline mutation in $E$-Cad strongly predisposes affected individuals to develop gastric cancer [17]. The first aim of this case-control study was therefore to investigate this hypothesis using single nucleotide polymorphisms (SNPs) in the genes of this ER-signaling pathway in order to define their tumorigenic contribution. We also included $M T A 1$, the other gene in the MTA family, in the study, as it regulates a pathway involved in blocking ER function $[12,18]$. Like MTA3, MTA1 can form a repressive chromatin complex [9]. The second aim was to determine whether the association between these pathways and breast cancer was modified by breast cancer risk factors reflecting estrogen exposure; if these susceptibility genes are associated with breast cancer development via the hypothesized mechanism involving estrogen, the relationship between cancer risk and susceptibility genotypes would be expected to be more significant in the subset of women with a longer period of estrogen exposure or higher estrogen levels.

\section{Materials and methods}

\section{Study population}

This case-control study is part of an ongoing cooperative study aimed at understanding the causes of breast cancer in Taiwan, which is characterized by low incidence [19], early tumor onset [20], hormone dependency [21, 22], and novel genomic alterations $[15,23]$. Because of the low incidence of breast cancer, which suggests an overall lower effect of common risk factors, and because of its homogenous genetic background, the Taiwanese population has certain advantages for studying the effects of subtle genetic variations, such as SNPs [22]. Furthermore, the use of a genetically homogenous population reduces the chance of false positives due to population stratification. The present study included 468 female breast cancer patients and 470 healthy female controls. All subjects gave their informed consent. 
All breast cancer patients had pathologically confirmed incident primary infiltrating ductal carcinoma of the breast (cases of lobular carcinomas were not included) and $6 \%$ had a family history of breast cancer (mother or sister). All were diagnosed and treated at the Tri-Service General Hospital between March 2002 and July 2004; these patients accounted for almost all (>90\%) women with breast cancer attending our breast cancer clinic during the study period, the remaining patients being excluded because of a lack of suitable blood specimens. No significant differences in breast cancer risk factors were found between the included and excluded women. More importantly, because the clinic in which this study was performed is one of the major breast cancer clinics in northern Taiwan, our patients accounted for a significant proportion (about 20\%) of all breast cancer cases diagnosed during the study period in this region.

To avoid any selection bias and differential recall bias of previous disease history, we purposely randomly selected the controls from women attending the health examination clinic of the same hospital during the same study period. These women underwent a 1-day comprehensive health examination (including regular breast screening using X-ray mammography and ultrasonic examination of the breasts) and any showing any evidence of breast cancer, suspicious precancerous lesions of the breast, or other cancers were excluded. These controls accounted for about $40 \%$ of all women attending the clinics and no significant differences were found in terms of socioeconomic status between those included and those not included.

The study protocol of the present study was approved by the institutional review broad of both Academia Sinica and the participating hospital. The consideration regarding methodological issues of the present study (such as study design, sampling scheme and potential bias) has been described in detail previously [21, 22, 24, 25]. The validity of our study approach has been also confirmed in these studies.

\section{Questionnaire}

An experienced research nurse was assigned to administer a structured questionnaire to both cases and controls. The validity of this questionnaire has been addressed and confirmed in our previous studies [21, 22, 24, 25]. The information collected included demographic characteristics (ethnic background, residence area, family income, and educational level), reproductive risk factors (age at menarche and/or menopause, age at FFTP, number of pregnancies, parity, history of breast feeding, and menopausal status), medical history [age at diagnosis of breast cancer, family history of breast cancer (first-degree relatives), history of breast biopsy, and history of breast screening], and exogenous hormone exposure [use of oral contraceptives and hormone replacement therapy (HRT)]. Body mass index (BMI) and a history of alcohol consumption or cigarette smoking were also recorded. Women younger than 55 years who had undergone hysterectomy, but not bilateral oophorectomy, were classified as unknown in terms of menopausal status.

\section{Genotyping}

Genomic DNA was extracted from the buffy coat isolated from whole blood samples using a QIAamp DNA extraction kit (Qiagen Inc.) following the manufacturer's protocol. Three SNPs for each of five genes in the ER-signaling pathways ( $E R$, MTA1, MTA3, Snail, and E-Cad) were selected and genotyped, the SNP information being obtained from the public SNP database maintained by the National Institute of Health, USA (website, http://www.ncbi.nlm.nih.gov/SNP/index.html). We chose SNP sites on the basis of at least one of the following requirements: (a) a genotype-phenotype association study had shown a defective or suboptimal function related to a specific allele [e.g. $C(-160) A$ and $G(-347) G A$ of $E-C a d][26,27]$; (b) previous epidemiological studies had demonstrated an association between a specific SNP and cancer risk (e.g. the SNPs of ER) [14]; and (c) an allele frequency of the minor allele greater than $5 \%$ had been reported in SNP databases. Since, with the exception of $G(-160) C$ and $G(-347) G A$ of $E-C a d$, there have not been any reports of an association between genotypes and phenotypic changes in these SNPs, these selected SNPs were used as markers to reflect possible linkage disequilibrium (LD) between themselves and different alleles a gene with an as yet undefined effect on phenotypic change.

All SNPs were genotyped using a highthroughput genotyping platform based on the 
$5^{\prime}$ nuclease allelic discrimination Taqman assay [28] in a 96-well format on the ABI Prism 7000HT Sequence Detection System (Applied Biosystems). The PCR primers and probes for individual SNPs were designed using the Assays-by-Design Service (Applied Biosystems). To ensure that the observed polymorphisms were specific and not the result of experimental variation, the results were confirmed by repeating $25 \%$ of the assays, and no inconsistent genotype was found.

\section{Statistical analysis}

We followed our previously established sequential steps for the statistical analysis of an association study [21, 22, 24, 25]:

(a) Univariate and multivariate analyses were used to determine risk factors and to establish background risk profiles for breast cancer in this series of study subjects. Though estrogen is a family of hormones, as our previous studies or many other studies [21, 22, 24, 25], important reproductive risk factors were used as indices to estimate the level of estrogen exposure or susceptibility to estrogen exposure in the subsequent analysis;

(b) To ensure that the controls used were representative of women in the general population and to exclude the possibility of genotyping error, deviation of the genotypic frequency of each SNP of individual genes in the control subjects from that expected under the Hardy-Weinberg equilibrium (HWE) was assessed using the goodness-offit test [29];

(c) The genotypic frequency of each SNP of individual genes was compared between cases and controls. Differences in genotypic frequency of individual SNPs between cases and controls were tested using multiple logistic regression models [30] with simultaneous consideration of known risk factors of breast cancer, and adjusted odds ratios for the association were estimated;

(d) The relationship between each gene and breast cancer risk in women with different levels of estrogen exposure or with different degrees of estrogen susceptibility was examined using the joint method [21, 22, 24, 25].
We calculated the risk of breast cancer associated with the combination of the putative high-risk genotype of each gene and a reproductive risk factor. Using $\beta$ estimates from the logistic regression model, in which we used a set of dummy variables [31] representing different combinations of gene (high-risk or low-risk genotype) and risk factor, we assessed the relative excess risk due to harboring a putative high-risk genotype within reproductive risk factor strata;

(e) Since low-penetrance alleles (e.g. SNPs) of cancer-associated genes in the same functional pathway can act in combination in a dosage-dependent manner to determine cancer predisposition [24, 32], a possible joint contribution of individual genes to increased breast cancer risk was explored. A set of dummy variables reflecting different combinations of genotypes of different genes in the same functional pathway was used in the logistic regression model. We also performed a conventional logistic regression, a test evaluating the statistical significance of a trend to an increase in the number of putative high-risk genotypes in all genes with increasing breast cancer risk. Furthermore, since MAT1 and MTA3 are part of different transcription suppressor complexes and interact differently with the ER [9, 12], we investigated separately the joint effect (reflected by the combinations of putative high-risk/ low-risk genotypes) of the genes participating in these subpathways, i.e. we looked separately for a joint effect of $E R, M T A 3$, Snail, and $E$-Cad and for a joint effect of $M T A 1$ and $E R$.

(f) Of particular interest was the relationship between the genes in the ER-signaling pathway and breast cancer risk in women with different levels of estrogen exposure or with different degrees of estrogen susceptibility, which was examined using joint and stratified methods [21]. In the joint method, we calculated the risk of breast cancer associated with the combination of the number of putative high-risk genotypes and a risk factor. In the stratified method, possible modification of risk associated with risk factor by ER-signaling genes was evaluated by calculating the $\mathrm{aOR}$ of breast cancer in relation 
to estrogen-related risk factors within different subgroups of women harboring different numbers of high-risk genotypes.

\section{Results \\ Risk factors profile}

The present study included 468 female patients with pathologically-confirmed infiltrating ductal carcinoma of the breast and 470 healthy female controls. The risk profile of this series of study subjects was similar to that reported in our previous breast cancer studies [21, 22, 24, 25], and a significantly increased risk was found to be conferred by a family history of breast cancer in female first-degree relatives (yes vs. no, adjusted odds ratio, aOR, 1.50; 95\% confidence interval, CI, 1.12-2.00). Of the various reproductive risk factors, pregnancy-related risk factors were consistently found to be highly associated with an increased risk. Using multiple logistic regression analysis, the cases were found to be younger at menarche than the controls, although the difference was not significant $(\leq 15$ years vs. $>15$ years, aOR, 1.15 ; 95\%CI, 0.79-1.67). However, compared to controls, the cases were significantly older at FFTP ( $>25$ years vs. $\leq 25$ years, aOR, 1.45; 95\% CI, 1.06-1.99), and the risk was significantly decreased by a greater number of fullterm pregnancies (FTPs) ( $\geq 3$ FTPs vs. no history of FTP, aOR, 0.60; 95\%CI, 0.37-0.97). These significant effects can be explained by the fact that FTP results in permanent differentiation of the vulnerable breast stem cells [33], altering subsequent susceptibility to hormones. No significant association was found between cancer risk and smoking status, radiation exposure, HRT, or dietary intake of specific kinds of foods or vegetables, but obese women showed a significantly increased risk (BMI $\geq 24 \mathrm{~kg} / \mathrm{m}^{2}$ vs. $<24 \mathrm{~kg} / \mathrm{m}^{2}$, aOR, 1.46; 95\%CI, 1.10-1.93). Obesity may lead to increased peripheral levels of estrogen, which is converted and released from adipose tissue by fat cells, which are rich in the enzyme, Aromatase, which catalyzes this conversion [34, 35]. This last fact is of particular relevance to the hypothesized mechanism in this study, as Aromatase expression can be specifically downregulated by Snail [11], thus linking obesity to the putative breast tumorigenic mechanism associated with one of the ERsignaling mechanisms addressed in the present study, i.e. the ER-MTA3-Snail-E-Cad pathway.

\section{Single SNP analysis of individual ER-signaling genes}

Fifteen SNPs of the 5 genes involved in the ERsignaling pathways (ER, MTA3, MTA1, Snail, and $E-C a d)$ were genotyped in an initial screening in 192 cases and 192 controls. Of these, 5 were not observed, and 3 were infrequent (frequency of the less frequent allele $<0.01$ ), so these 8 were not genotyped in the remainder of the samples. The remaining 7 SNPs (2 for $E R, 1$ for $M T A 3,1$ for Snail, 2 for $E-C a d$, and 1 for $M T A 1)$ were genotyped in all cases and controls. All 7 were in HWE in the controls, thus excluding the possibility of genotyping error. Since the $2 E R$ SNPs were in strong $\operatorname{LD}(p<0.01)$ in both cases and controls, as were the 2 E-Cad SNPS $(p<0.01)$, and since the frequency distribution based on haplotypes was similar to that based on individual SNPs, we chose the SNP which showed the most significant $p$ value in the multivariate logistic regression analyses to represent the allelic status of each of these two genes. Furthermore, in the subsequent analyses, in order to look for an association between breast cancer risk and genotypic polymorphism, we defined the susceptibility (high-risk) alleles of $E R$, $M T A 3$, Snail, and MTAl on the basis of the findings of the present study. This definition is genetically reasonable, since possible LD between these SNPs and functionally significant alleles is expected to differ in different populations [24]. In addition, since functional assay has shown that the $C(-160) A$ variant allele of $E-C a d$ is associated with reduced expression and, thus, suboptimal function of E-Cad [26, 27], this variant allele has also been defined as a putative high-risk allele. To explore a possible association between breast cancer and individual SNPs of the five genes, the heterozygous and homozygous variant genotypes were grouped together and compared to the homozygous wild-type genotype (Table 1) except in the case of MTA3 (see below). Because of the small percentage of individuals among our subjects with the homozygous variant genotype, this grouping resulted in increased statistical power in detecting the main effect. In the case of $M T A 3$, 
breast cancer risk was lower in women harboring the heterozygous variant genotype than in those with the homozygous wild-type genotype, and it was therefore more appropriate to group together the heterozygous and homozygous wild-type genotypes and compare these to the homozygous variant genotype (Table 1). When the genotype distributions of the five genes were compared between cases and controls and the effects of breast cancer risk factors simultaneously adjusted in the multivariate logistic regression models, the SNP in E-Cad was found to reach statistical significance $(p=0.04)$ (Table 1).

The joint effect of SNP of ER-signaling genes and reproductive risk factors

As we have shown previously [22, 24, 25], in the case of an effect contributed by a low-penetrance allele, it is not unusual to observe only a minor increase in risk associated with putative high-risk genotypes, and the effects of these low-penetrance genotypes only become obvious in the presence of exogenous and endogenous risk factors. Because the ER-MTA3-Snail-E-Cad pathway or ER$M T A 1$ are activated in response to estrogen signaling [8, 11, 12], it is tempting to speculate that these susceptibility genotypes would act jointly with estrogen-related risk factors to increase breast cancer risk. To explore this possibility, we therefore investigated whether the relationship between cancer risk and susceptibility genotypes was more significant in the subset of women expected to have been more exposed to estrogen (obese) or to be more susceptible to estrogen (older age at FFTP) (Table 2). For the analysis, the reference group consisted of women with no putative high-risk genotype and having

Table 1. Genotype frequencies in breast cancer cases and controls of sequence variants (single nucleotide polymorphism, SNPs) of the five genes [estrogen receptor (ER), Metastasis-associated 3 (MTA3), Snail, E-Cadherin (E-Cad), and Metastasis-associated 1 $(M T A 1)]$ involved in ER-signaling pathways and the adjusted odds ratio (aOR) for breast cancer risk.

\begin{tabular}{|c|c|c|c|}
\hline SNP and genotype ${ }^{a}$ & No. of cases $(\%)$ & No. of controls $(\%)$ & $\operatorname{aOR}(95 \% \mathrm{CI})^{\mathrm{b}}$ \\
\hline \multicolumn{4}{|c|}{$E R, G 2142 A, T h r 594 T h r,(\operatorname{rs} 2228480)$} \\
\hline$G G$ & $260(55.7)$ & $282(60.3)$ & \multirow{3}{*}{$\begin{array}{l}1.00 \text { (Ref. })^{\mathrm{c}} \\
1.27(0.95-1.70\end{array}$} \\
\hline$G A$ & $182(38.9)$ & $159(33.3)$ & \\
\hline$A A$ & $25(5.4)$ & $27(5.8)$ & \\
\hline \multicolumn{4}{|c|}{ MTA3, T149529C, (rs2272447) } \\
\hline$T T$ & $151(32.3)$ & $156(33.2)$ & \multirow{2}{*}{1.00 (Ref.) } \\
\hline$T C$ & $219(46.8)$ & 233(49.6) & \\
\hline$C C$ & $98(20.9)$ & $81(17.2)$ & $1.36(0.97-1.93)$ \\
\hline \multicolumn{4}{|c|}{ Snail, T1171C, Val118Ala, (rs4647958) } \\
\hline $\mathrm{Val} / \mathrm{Val}$ & $375(80.3)$ & $374(79.7)$ & $1.00(\text { Ref. })^{\mathrm{c}}$ \\
\hline Val/Ala & $87(18.6)$ & $93(19.8)$ & \multirow{2}{*}{$0.94(0.68-1.31)$} \\
\hline Ala $/$ Ala & $5(1.1)$ & $2(0.4)$ & \\
\hline \multicolumn{4}{|c|}{$E-C a d, C-160 A,(\operatorname{rs} 16260)$} \\
\hline$C C$ & $222(47.5)$ & $243(51.8)$ & $1.00(\text { Ref. })^{\mathrm{c}}$ \\
\hline$C A$ & $201(43.0)$ & 187(39.9) & \multirow{2}{*}{$1.31(1.01-1.71)$} \\
\hline$A A$ & $44(9.4)$ & $39(8.3)$ & \\
\hline \multicolumn{4}{|c|}{$M T A 1, A 1121 G,(\mathrm{rs} 4983413)$} \\
\hline$A A$ & $215(46.0)$ & $194(54.3)$ & $1.20(0.92-1.58)$ \\
\hline$A G$ & $204(43.7)$ & $222(45.7)$ & \multirow{2}{*}{1.00 (Ref.) $)^{\mathrm{c}}$} \\
\hline$G G$ & $48(10.3)$ & $52(11.1)$ & \\
\hline
\end{tabular}

${ }^{\mathrm{a}}$ The numbers represent the position (measured in base pairs) from the transcription site and the letters the nucleotide changes. If the SNP is in an exon, the position of the amino acid is also given. The number shown in parenthesis is the NCBI SNP cluster ID for each SNP.

${ }^{\mathrm{b}}$ The adjusted odds ratio (aOR) and $95 \%$ confidence interval $(95 \% \mathrm{CI})$ were estimated in logistic regression models containing breast cancer risk factors, including age, a family history of breast cancer, a history of full-term pregnancy, and the body mass index. The minor difference in sample size in individual comparisons was due to a lack of DNA specimens for study subjects.

${ }^{\mathrm{c}}$ Ref, reference group. 
Table 2. Risk (adjusted odds ratio, aOR) of breast cancer associated with the combination (joint effect) of putative high-risk genotypes of the genes involved in estrogen receptor (ER)-signaling pathways and a risk factor [age at first full-term pregnancy (FFTP)] or obesity, defined as a body mass index (BMI) $\geq 24 \mathrm{~kg} / \mathrm{m}^{2}$.

\begin{tabular}{|c|c|c|c|c|}
\hline Genotype of ER-signaling genes & Age at FFTP (years) & $\mathrm{aOR}(95 \% \mathrm{CI})^{\mathrm{a}}$ & BMI, $\mathrm{kg} / \mathrm{m}^{2}$ & $\mathrm{aOR}(95 \% \mathrm{CI})^{\mathrm{a}}$ \\
\hline \multicolumn{5}{|l|}{ ER, Thr594Thr, G2142A } \\
\hline$G G$ & $<26$ & $1.00(\text { Ref. })^{\mathrm{b}}$ & $<24$ & $1.00(\text { Ref. })^{b}$ \\
\hline$G A, A A$ & $<26$ & $1.41(0.92-2.15)$ & $<24$ & $1.30(0.92-1.84)$ \\
\hline$G G$ & $\geq 26$ & $1.59(1.07-2.36)$ & $\geq 24$ & $1.45(0.99-2.11)$ \\
\hline$G A, A A$ & $\geq 26$ & $1.79(1.16-2.76)$ & $\geq 24$ & $1.64(1.08-2.48)$ \\
\hline$p$ for trend ${ }^{\mathrm{c}}$ & \multicolumn{2}{|c|}{0.007} & \multicolumn{2}{|c|}{0.01} \\
\hline \multicolumn{5}{|l|}{ МТАЗ, T149529C } \\
\hline$T T, T C$ & $<26$ & $1.00(\text { Ref. })^{b}$ & $<24$ & $1.00(\text { Ref. })^{b}$ \\
\hline$C C$ & $<26$ & $1.22(0.71-2.09)$ & $<24$ & $1.30(0.81-1.88)$ \\
\hline$T T, T C$ & $\geq 26$ & $1.39(0.99-1.94)$ & $\geq 24$ & $1.45(0.95-1.78)$ \\
\hline$C C$ & $\geq 26$ & $1.72(1.01-2.91)$ & $\geq 24$ & $2.19(1.19-4.01)$ \\
\hline$p$ for trend ${ }^{\mathrm{c}}$ & \multicolumn{2}{|c|}{0.02} & \multicolumn{2}{|c|}{0.007} \\
\hline \multicolumn{5}{|l|}{$E-C a d, C-160 A$} \\
\hline$C C$ & $<26$ & $1.00(\text { Ref. })^{b}$ & $<24$ & $1.00(\text { Ref. })^{b}$ \\
\hline$C A, A A$ & $<26$ & $1.32(0.87-2.00)$ & $<24$ & $1.09(0.76-1.57)$ \\
\hline$C C$ & $\geq 26$ & $1.51(0.99-2.30)$ & $\geq 24$ & $1.01(0.67-1.55)$ \\
\hline$C A, A A$ & $\geq 26$ & $1.82(1.19-2.79)$ & $\geq 24$ & $1.61(1.06-2.46)$ \\
\hline$p$ for trend $d^{\mathrm{c}}$ & \multicolumn{2}{|c|}{0.007} & \multicolumn{2}{|c|}{0.04} \\
\hline \multicolumn{5}{|l|}{$M T A 1, A 1121 G$} \\
\hline$A G, G G$ & $<26$ & $1.00(\text { Ref. })^{b}$ & $<24$ & $1.00(\text { Ref. })^{b}$ \\
\hline$A A$ & $<26$ & $1.18(0.78-1.79)$ & $<24$ & $1.11(0.79-1.56)$ \\
\hline$A G, G G$ & $\geq 26$ & $1.47(0.98-2.21)$ & $\geq 24$ & $1.26(0.86-1.86)$ \\
\hline$A A$ & $\geq 26$ & $1.71(1.10-2.65)$ & $\geq 24$ & $1.67(1.11-2.51)$ \\
\hline$p$ for trend $d^{\mathrm{c}}$ & \multicolumn{2}{|c|}{0.02} & \multicolumn{2}{|c|}{0.01} \\
\hline \multicolumn{5}{|l|}{ Snail, T1171C, Val118Ala } \\
\hline $\mathrm{Val} / \mathrm{Val}$ & $<26$ & $1.00(\text { Ref. })^{\mathrm{b}}$ & $<24$ & $1.00(\text { Ref. })^{b}$ \\
\hline Val $/$ Ala, Ala $/$ Ala & $<26$ & $1.86(1.07-3.23)$ & $<24$ & $1.05(0.69-1.58)$ \\
\hline Val $/$ Val & $\geq 26$ & $1.79(1.27-2.52)$ & $\geq 24$ & $1.42(1.03-1.95)$ \\
\hline Val $/$ Ala, Ala $/$ Ala & $\geq 26$ & $0.94(0.56-1.59)$ & $\geq 24$ & $1.17(0.66-2.09)$ \\
\hline$p$ for trend $d^{c}$ & \multicolumn{2}{|c|}{0.17} & \multicolumn{2}{|c|}{0.15} \\
\hline
\end{tabular}

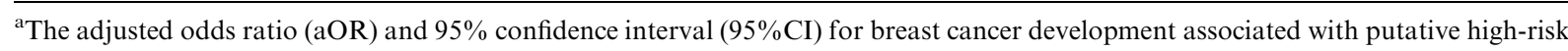
genotypes and risk factors were estimated in a multivariate logistic regression model containing age, family history of breast cancer, and a group of dummy variables to represent the four different combinations of genotype and risk factor status.

${ }^{\mathrm{b}}$ Ref, reference group.

${ }^{\mathrm{c}} \mathrm{A}$ logistic regression was performed to test whether a trend to an increase in the composite variable (a continuous term) combining both the genotype and risk factor with increasing breast cancer risk (measured by the $\beta$ estimates from this regression model) was statistically significant.

been less exposed to estrogen or being less susceptible to estrogen exposure (with a lower BMI or younger at FFTP). Our hypothesis was supported by the finding that, in the absence of the estrogenrelated risk factors, the harboring of putative highrisk genotypes was associated with a smaller, nonsignificant increase in risk, whereas, in the presence of these risk factors, the harboring of putative high-risk genotype was associated with a much greater and significant combined risk of breast cancer (Table 2). Furthermore, the presence of a joint effect of high-risk genotypes and risk factors was examined in a logistic regression model, in which tests for trend were done by treating each categorized variable as a composite variable (a continuous term) used to reflect different combinations of risk 
Table 3. Risk (adjusted odds ratio, aOR) of breast cancer development associated with genotypic polymorphism of Snail, stratified by the reproductive risk factor, age at firstfull-term pregnancy (FFTP).

\begin{tabular}{lll}
\hline Age at FFTP & $\begin{array}{l}\text { Genotype of } \\
\text { Snail T1171C, }\end{array}$ & \\
& $\mathrm{aOR}^{\mathrm{a}}(95 \% \mathrm{CI})$ \\
& Vall l8Ala & \\
\hline$<26$ years & $<$ Val $/$ Val & $1.00(\text { Ref. })^{\mathrm{b}}$ \\
& Val $/$ Ala, Ala $/$ Ala & $1.92(1.15-3.20)$ \\
$\geq 26$ years & Val $/$ Val & $1.00(\text { Ref. })^{\mathrm{b}}$ \\
& Val $/$ Ala,Ala $/$ Ala & $0.54(0.34-0.84)$ \\
\hline
\end{tabular}

${ }^{\text {a }}$ The adjusted odds ratio (aOR) and $95 \%$ confidence interval $(95 \% \mathrm{CI})$ for breast cancer development were estimated in a multivariate logistic regression model containing age, a family history of breast cancer, and Snail genotypic polymorphism. ${ }^{\mathrm{b}}$ Ref, reference group.

factors and genotype. Having a higher number of composite variables was found to be significantly associated with an increase in breast cancer risk ( $p$ for trend $<0.05$ ) (Table 2), the only exception, interestingly, being the combination of Snail and estrogen-related risk factors, with no increase in aOR for the group of women with the high-risk genotype of Snail and who were older at FFTP or obese (Table 2). To explore this finding, we stratified our study participants on the basis of age at FFTP and found that a significant increase in cancer risk associated with high-risk genotypes of Snail was only seen in women having their FFTP at a younger age; in contrast, in the group of women who were older at FFTP, the putative high-risk genotypes of Snail were associated with a significant decrease in cancer risk (Table 3). This gene was therefore not included in the subsequent analysis.

\section{The joint effect of genes participating in common ER-signaling pathways}

Given that individual genes in ER-MTA3-E-Cad pathway and $E R-M T A 1$ pathway were found to participate cooperatively in response to estrogen signaling and given that an increased risk of cancer due to a combined effect of genes belonging to a common tumorigenic pathway has been demonstrated in a mouse model [36], cell-line-based functional studies [32] and epidemiological findings [21, 22, 24], we examined whether a joint effect of these genes was associated with breast cancer

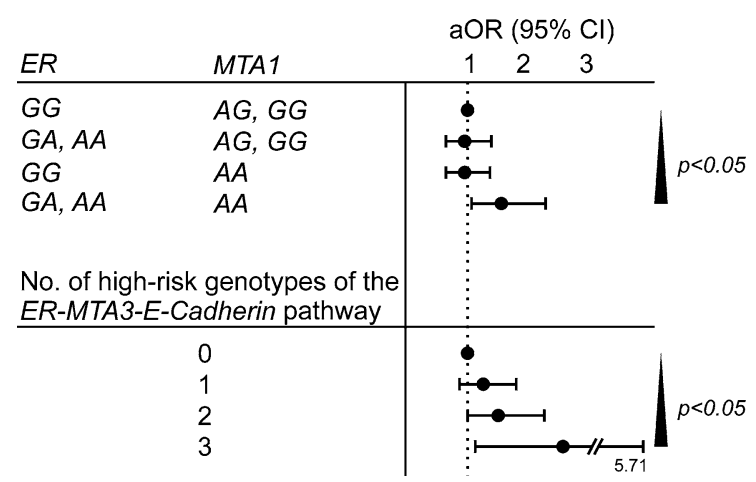

Figure 1. Risk (adjusted odds ratio, aOR) of breast cancer development associated with a joint effect of genotypic polymorphism of the genes in each of the different estrogen-receptor (ER) signaling subpathways. The adjusted odds ratio $(\mathrm{aOR})$ and $95 \%$ confidence interval $(95 \% \mathrm{CI})$ for breast cancer development were estimated in a multivariate logistic regression model containing age, family history of breast cancer, reproductive risk factors, and a set of dummy variables representing different combinations of genotypes. A logistic regression was performed to test whether a trend to an increase in the number of putative high-risk genotypes with increasing breast cancer risk (measured by the $\beta$ estimate from this regression model) was statistically significant (indicated by the $p$ value for trend).

development by determining the breast cancer risk associated with harboring different numbers of putative high-risk genotypes (Figure 1). To exclude a false combination effect due to an unequal contribution of individual genes, using a dummy variable coding scheme and women with all putative low-risk genotypes as the reference group, we separately estimated the risk associated with harboring different numbers of putative highrisk genotypes. Based on the suggested mechanisms of ER-signaling [8, 12, 18], two subpathways (ER-MTA3-E-Cad and ER-MTA1) were specified, and we looked for a possible joint effect among genes within each subpathway. The results were consistent with the presence of a joint effect, a higher risk being seen in women harboring a higher number of high-risk genotypes (Figure 1). It should be noted that, although only E-Cad genotypic polymorphism was significantly associated with breast cancer in the single gene analysis (Table 1), the joint effect analysis suggested that the genes in the same functional pathways act together to increase the risk of breast cancer, which is in line with a joint effect of individual genes in these subpathways being related to breast cancer development. 
The joint effect of ER signaling pathways and reproductive risk factors

In the above analyses, we separately demonstrated (i) that a greater effect was seen with combinations of high-risk genotypes of individual genes and reproductive risk factors (Table 2) and (ii) that there was a joint effect of genes in the same mechanistic subpathway (Figure 1). Thus, it is tempting to consider these two findings together and to speculate that, if these subpathways were involved in breast tumorigenesis via its response to estrogen signaling, the relationship between breast cancer risk and reproductive risk factor would not be the same in women harboring different high-risk genotypes of individual functional subpathways. This was evaluated by calculating the risk (aOR) of breast cancer associated with the joint effect of obesity and a higher number of high-risk genotypes in individual functional subpathways (Table 4). We first classified the women into two groups with a lower or higher number of putative high-risk genotypes, since such a definition would give sufficient statistical power to address the question. The reference group consisted of women who had a lower number of putative high-risk genotypes and were not obese. Our hypothesis was supported by the finding that, in the absence of obesity, the harboring of a greater number of putative high-risk genotypes was associated with a small, non-significant increase in risk, whereas, in obese women, it was associated with a much greater and more significant combined risk of breast cancer (Table 4). It is notable that, in terms of the individual subpathways, the joint effect associated with obesity and ER-MTA3-E$C a d$ was stronger than that seen with the $E R-M T A 1$, and the increase in breast cancer risk more than additive.

Finally, to confirm the joint effect between genotypic polymorphism and obesity seen above, we estimated the risk associated with obesity in women with different numbers of high-risk genotypes of individual subpathways. The expected potential importance of a risk effect conferred by obesity was observed, but modification of this risk effect by genotypic polymorphism was supported by the findings shown in Table 5. In the "lower number of putative highrisk genotypes" strata, there was only a minor,
Table 4. Risk (adjusted odds ratio, aOR) of breast cancer associated with the combination (joint effect) of the number of putative high-risk genotypes of the genes involved in different estrogen receptor (ER)-signaling pathways and obesity, defined as a body mass index (BMI) $\geq 24 \mathrm{~kg} / \mathrm{m}^{2}$.

\begin{tabular}{lcc}
\hline $\begin{array}{l}\text { ER-signaling } \\
\text { subpathway/Number } \\
\text { of putative high-risk } \\
\text { genotypes }\end{array}$ & $\mathrm{BMI}, \mathrm{kg} / \mathrm{m}^{2}$ & $\mathrm{aOR}^{\mathrm{a}}(95 \% \mathrm{CI})$ \\
\hline $\begin{array}{l}\text { ER-MTA1 } \\
0\end{array}$ & $<24$ & $1.00(\text { Ref. })^{\mathrm{b}}$ \\
$1-2$ & $\geq 24$ & $1.12(0.79-1.59)$ \\
0 & $<24$ & $1.50(0.93-2.44)$ \\
$1-2$ & $\geq 24$ & $1.63(1.10-2.42)$ \\
& & $p$ for trend $\mathrm{d}^{\mathrm{c}}<0.05$ \\
ER-MTA3-E-Cad & $<24$ & $1.00(\text { Ref. })^{\mathrm{b}}$ \\
$0-1$ & $\geq 24$ & $1.19(0.84-1.67)$ \\
$2-3$ & $<24$ & $1.22(0.85-1.76)$ \\
$0-1$ & $\geq 24$ & $2.37(1.47-3.38)$ \\
$2-3$ & & $p$ for trend ${ }^{\mathrm{c}}<0.05$ \\
\hline
\end{tabular}

${ }^{a}$ The adjusted odds ratio (aOR) and $95 \%$ confidence interval $(95 \% \mathrm{CI})$ for breast cancer development were estimated in a multivariate logistic regression model containing age, family history of breast cancer, and a set of dummy variable representing different combinations of genotype and risk factor.

${ }^{\mathrm{b}}$ Ref, reference group.

${ }^{\mathrm{c}} \mathrm{A}$ logistic regression was performed to test whether a trend to an increase in the composite variable (a continuous term) combining both the number of putative high-risk genotypes and risk factors with increasing breast cancer risk (measured by the $\beta$ estimates from this regression model) was statistically significant.

Table 5. Risk (adjusted odds ratio, aOR) of breast cancer development associated with obesity, defined by a body mass index (BMI) $\geq 24 \mathrm{~kg} / \mathrm{m}^{2}$, stratified by the number of high-risk genotypes of the genes involved in estrogen-receptor(ER)signaling pathways.

\begin{tabular}{llll}
\hline $\begin{array}{l}\text { ER-signaling } \\
\text { subpathway/ }\end{array}$ & \multicolumn{3}{l}{$\mathrm{BMI} \geq 24 \mathrm{~kg} / \mathrm{m}^{2}$} \\
$\begin{array}{l}\text { Case } \\
\text { Number of } \\
\text { high-risk } \\
\text { genotypes }\end{array}$ & $\begin{array}{l}\text { Control } \\
(\%)\end{array}$ & $\begin{array}{l}\mathrm{aOR}^{\mathrm{a}} \\
(95 \% \mathrm{CI})\end{array}$ \\
\hline ER-MTA1 & & & \\
0 & $73(40.8)$ & $68(33.3)$ & $1.34(0.80-2.22)$ \\
$1-2$ & $132(47.3)$ & $73(27.9)$ & $1.52(1.08-2.15)$ \\
ER-MTA3-E-Cad & & \\
$0-1$ & $132(43.9)$ & $108(32.4)$ & $1.24(0.81-1.90)$ \\
$2-3$ & $73(46.5)$ & $32(24.2)$ & $2.29(1.35-3.89)$ \\
\hline
\end{tabular}

a The adjusted odds ratio (aOR) and $95 \%$ confidence interval $(95 \% \mathrm{CI})$ for breast cancer development were estimated in a multivariate logistic regression model containing age, family history of breast cancers, reproductive risk factors and BMI. 
non-significant difference in risk of developing breast cancer associated with obesity. However, in the women with a higher number of putative high-risk genotypes, a significant increase was seen with obesity.

\section{Discussion}

\section{Methodological consideration}

This genotype-based association study provides evidence for the tumorigenic contribution of two specific subpathways involved in ER-signaling mechanism and for high-risk genotypes of the genes in the ER-MTA3-E-Cad and ER-MTAI increasing breast cancer risk. This was addressed in a multigenic model, and possible modifications due to known risk factors, including estrogen exposure and BMI, were also considered. Since the present study used a candidate gene approach based on SNPs located in the genes of the ERsignaling pathway known to be causally linked to cell outgrowth and increased invasiveness [8, 11, 12], the findings are mechanistically plausible. However, as mentioned previously [24, 25], one of the most important issues in using SNPs in an association study is the interpretation of the identified association between SNPs and a given trait. Since most of the SNPs analyzed in our multivariate models are not known to affect protein function, the observed associations between breast cancer risk and SNPs should be interpreted as the presence of LD between these SNPs and other SNPs in exons resulting in functional polymorphism. We considered that single SNPs may fail to capture all of the contribution of a locus to a particular trait and therefore used more than one SNP in these genes to assign the haplotypes and to examine haplotype effects on cancer risk, but the information generated by haplotype analysis was limited, due to strong LD between SNPs in the same gene. However, these SNPs, which probably have no functional effect, may be of particular methodological importance and advantage in addressing the tumorigenic contribution of the ER-signaling pathway to breast cancer risk. The reason for this is that, although ER-MTA3-E-Cad and ER-MTAI are essential for regulating ER signaling in the normal and malignant mammary epithelium $[8,11,12,18]$, specific alleles of these genes can intrinsically be associated with both a tumor-promoting and an anti-tumor mechanism because of the stage-related changes in the tumorigenic effects contributed by $E R / E-C a d[11,14,16]$ and the complexity of the chromatin-remodeling mechanisms involving MTA proteins during tumor initiation, promotion, and progression [9]. These paradoxically different roles of these ER-signaling genes strongly suggest that the balance and interaction between all the genes in the mechanistic pathway may be as important as the individual genes. Thus, the use of SNPs with no functional effects enabled us to examine putative associations without resorting to an a priori hypothesis that a decreased ER-MTA3$E$-Cad or ER-MTAl capacity was related to an increased risk of cancer.

The present study did not exclude any subjects having a family history of breast cancer from the control group. Because family history of breast cancer is one of the well-established risk factors for breast cancer, the major epidemiological consideration, if any, of the present study, would be some of the control subjects might develop breast cancer later. Thus, to address possible confounding effects due to this risk factor, we have included it in all multivariate analysis. In addition, if the genes involved in the estrogen-receptor-signaling pathway was associated with breast cancer risk as suggested, breast cancer predisposition due to SNPs of these genes might be reflected by having a positive family history of breast cancer in the controls as well as the cases. As a result, without exclusion of the women having a family history of breast cancer from our controls might lead to underestimation of genetic effect, and our estimation of risk associated with SNPs would be conservative.

\section{ER in association with breast cancer risk}

Reproductive hormones, particularly estrogen, play a critical role in breast cancer etiology [1-4]. On the basis of the well-known mechanism that the effect of estrogen is mediated primarily through high-affinity binding to the ER [5], some epidemiological studies have evaluated the association between genetic polymorphisms in $E R$ and breast cancer risk (e.g. Ref. [14]). The present study tested the breast tumorigenic contribution of the $E R$ genotype using a different approach, in 
which we examined whether breast tumorigenesis was linked to genotypes of candidate genes in the ER signal transduction pathway, and our findings provide further insight into the $E R$-associated pathogenesis of breast cancer development. At first glance, our data do not seem to totally support a breast tumorigenic contribution of the SNP of $E R$, since the genotype distribution was not significantly different between our breast cancer patients and controls (Table 1). However, our observation that specific combinations of variant genotypes of the genes in specific molecular pathways, in particular, the ER-MTA3-E-Cad pathway, were more common in patients than controls (Figure 1) prompts us to favor the interesting possibility that the SNP of $E R$ is associated with breast cancer risk, but this can only be seen in the subgroup of women harboring variant genotypes of MTA3 and E-Cad. Given this, the discrepancy about the degree and nature of cancer risk related to various genetic polymorphisms of $E R$ seen in previous studies is not surprising. To the best of our knowledge, this is the first study to address the issue of $E R$ polymorphism in relation to breast cancer risk in a multigenic model. The strength of this strategy should allow a more precise evaluation of the risks associated with $E R$ and a more comprehensive insight into breast tumorigenesis caused by estrogen exposure.

\section{Chromatin remodeling and cancer}

Appropriate regulation of gene expression requires an interplay of the complexes that remodel chromatin structure and thus regulate the accessibility of individual genes to specific transcription factors and the transcription machinery [9, 37]. It is therefore not surprising that tumor development, during which many genes are aberrantly expressed, is associated with chromatin-remodeling mechanisms, and that deficiency of chromatinremodeling complex components causes cancer. A good example is that mutation and homozygous deletion of the $h S N F 5 / I N I 1$ gene, which encodes a member of the chromatin-remodeling SWI/SNF multiprotein complexes, have been found in malignant rhabdoid tumors, extremely aggressive cancers of early childhood, which occur in various locations, mainly the kidney, brain, and soft tissues [38]. However, given the emerging important role of the chromatin-remodeling mechanisms in cancer formation, it is puzzling that genetic evidence linking common cancers and mutated chromatin-remodeling genes is very rare. The frequency of somatic mutations in the chromatin-remodeling genes in common cancers is very low $[39,40]$. The lack of such an association prevents any conclusion about the role of these genes in human cancer development to be drawn. We previously proposed a possible explanation for these paradoxes and suggested that, since the chromatin-remodeling genes are crucial for cells to maintain stability of chromatin structure and normal gene expression, any severe defects in them (e.g., mutation) would result in disruption of expression regulation of many genes, which would then trigger cell cycle checkpoint surveillance, leading to cell death [24]. Consistent with this expectation, in a mouse model, homozygous deficiency of $\operatorname{Snf5} 5$ results in early embryonic death [37]. The present study, which demonstrates an association between breast cancer risk and SNPs (the most frequent and most subtle genetic variation in the human genome) in MTA1 and MTA3, supports the possibility that subtle defects in these genes arising from low-penetrance (risk) alleles (e.g., hypomorphic mutants or polymorphic variants) might be able to escape checkpoint surveillance and lead to subtly aberrant expression of cancer-associated genes required for tumor formation. This "hide-then-hit" model [24] to explain the tumorigenic effect contributed by mutator genes, defects of which do not directly affect the rate of cell growth or death, but increase the chance of mutation and abnormal expression of oncogenes and tumor suppressor genes, is supported by our previous studies [24, 25, 41] showing that SNPs of DNA double-strand-break repair genes and mitosis-regulating genes are associated with breast cancer development. However, since the effects of SNP are low-penetrant, these variant alleles would predispose carriers to a higher risk of developing cancer, but not necessarily cause cancer. The probability of manifesting the tumorigenic phenotype depends on the joint effect between these alleles and other functionallyrelated alleles or the environment. This may explain why we observed a significant joint effect on breast cancer risk between MTAgenes and either other genes in the ER-signaling pathway or reproductive risk factors reflecting estrogen exposure or obesity. 


\section{Tumorigenic contribution of E-Cadherin}

The multigenic approach used in this study provided a unique opportunity to evaluate the relative importance of individual genes in the ER-signaling pathways in breast cancer development, and led to $E$-Cad being identified as the most important gene. Because E-Cad codes for a cell-cell adhesion molecule implicated in metastasis suppression [42], the reasons why it is the most important gene in this pathway predisposing to an increased risk of breast cancer initiation are therefore of interest. It is likely that suboptimal regulation of normal cell-cell adhesion due to decreased E-Cad expression resulting from harboring variant genotypes of $E$-cad plays a role in the initiation of cell proliferation by allowing escape from growth-control signals [17]. Alternatively, the cytoplasmic domain of E-Cad may modulate the Wnt signaling pathway by decreasing the amount of free cytoplasmic $\beta$-catenin entering the nucleus and triggering the expression of the positive cell-cycle regulator, cyclin D1, a mechanism also leading to cell outgrowth and tumor initiation [43, 44].

\section{Complexity of Snail in breast cancer development}

The finding that genotypic polymorphism of Snail interacts differently with reproductive risk factors in relation to breast cancer risk is of particular significance. This emphasizes the complexity of the molecular function of Snail in breast epithelium, and might, at least in part, reflect the downstream molecules with which Snail interacts in breast cells in women with different degrees of susceptibility to estrogen exposure. Snail, as a transcriptional repressor, suppresses the expression of E-Cad [10], thus resulting in increased breast cancer risk because of the tumor suppressor function of E-Cad; on the other hand, Snail also suppresses the expression of Aromatase [12, 45], decreased activity of which leads to decreased peripheral levels of estrogen, converted from adipose tissue, and thus to protection from breast cancer. Interestingly, our findings suggest the relative importance in breast cancer development of Snail-E-Cad (examined in this paper) and Snail-Aromatase (not examined directly) is not the same in women who have their FFTP at a younger or older age, respectively. Experimental studies in rats have shown that FTP results in permanent differentia- tion of the vulnerable breast stem cells, altering subsequent susceptibility to hormones [33]; this suggests that the age at FFTP may also be critical. In women having their FFTP at an older age and whose breast cells are therefore exposed to estrogen for a longer period, the tumorigenic potential associated with Aromatase and peripheral circulating estrogen will become obvious, and the transcriptional suppression of Aromatase by Snail is seen to be protective against breast cancer. In contrast, in women having their FFTP at a younger age, the tumorigenic potential directly associated with the cumulative estrogenic effect may not be as significant as that in women of an older age, and, consequently, the increased breast cancer risk associated with harboring variant genotypes of Snail, which has been shown in a cell line study to suppress E-Cad expression [10], would become more critical. Although the mechanism underlying this association and interaction has yet to be defined, the finding that genotypic polymorphisms of Snail interact differently with a estrogen-related risk factor in relation to breast cancer risk (Table 3) clearly demonstrates that the "dosage" of agents targeted genes (i.e. estrogen, reflected in the present study by the reproductive risk factors) might affect the association of cancers with polymorphisms of individual genes in different study groups.

\section{Conclusion}

Taken together, our epidemiological findings highlight the role of newly identified novel ER-signaling pathways relevant to breast cancer development and provide a more comprehensive picture of the tumorigenic effect of estrogen in breast cancer development. However, the positive association results between genotypic polymorphism of these genes and breast cancer risk wee only observed in combination with risk factors of breast cancer or in the subgroup of women subjects, not in single SNP analysis. Also, most significant findings were obtained from the results of trend test, not from individual categories. Thus, the genetic association evidence between the estrogen-receptor-signaling pathway and breast cancer should be interpreted with caution. Furthermore, there has been little research regarding these associations addressed in the present study, and therefore, the identified associations regarding the ER-signaling pathways 
with breast cancer can be viewed as important and exploratory clues for further experimental or epidemiological studies to understand the role of estrogen or reproductive risk factors during breast tumorigenesis.

\section{References}

1. Hulka B.S. and Stark A.T., Breast cancer: cause and prevention. Lancet 346: 883-887, 1995.

2. Kelsey J.L., Gammon M.D. and John E.M., Reproductive factors and breast cancer. Epidemiol. Rev. 15: 36-47, 1993.

3. Pike M.C., Spicer D.V., Dahmoush L. and Press M.F., Estrogens, progestogens, normal breast cell proliferation, and breast cancer risk. Epidemiol. Rev. 15: 17-35, 1993.

4. Gruber C.J., Tschugguel W., Schneeberger C. and Huber J.C., Production and actions of estrogens. N. Engl. J. Med. 346: 340-352, 2002.

5. Dickson R.B. and Stancel G.M., Estrogen receptor-mediated processes in normal and cancer cells. J. Natl. Cancer Inst. Monogr. 27: 135-145, 2000.

6. Frasor J., Danes J.M., Komm B., Chang K.C., Lyttle C.R. and Katzenellenbogen B.S., Profiling of estrogen up- and down-regulated gene expression in human breast cancer cells: insights into gene networks and pathways underlying estrogenic control of proliferation and cell phenotype. Endocrinology 144: 4562-4574, 2003.

7. Fujita N., Kajita M., Taysavang P. and Wade P.A., Hormonal regulation of metastasis-associated protein 3 transcription in breast cancer cells. Mol. Endocrinol. 18: 2937-2949, 2004.

8. Fujita N., Jaye D.L., Kajita M., Geigerman C., Moreno C.S. and Wade P.A., MTA3, a Mi-2/NuRD complex subunit, regulates an invasive growth pathway in breast cancer. Cell 113: 207-219, 2003.

9. Kumar R., Wang R.A. and Bagheri-Yarmand R., Emerging roles of MTA family members in human cancers. Semin. Oncol. 30((5 Suppl 16)) 30-37, 2003.

10. Batlle E., Sancho E., Franci C., Dominguez D., Monfar M., Baulida J. and Garcia De Herreros A., The transcription factor snail is a repressor of E-cadherin gene expression in epithelial tumour cells. Nat. Cell Biol. 2: 84-89, 2000.

11. Fearon E.R., Connecting estrogen receptor function, transcriptional repression, and E-cadherin expression in breast cancer. Cancer Cell 3: 307-310, 2003.

12. Kumar R., Another tie that binds the MTA family to breast cancer. Cell 113: 142-143, 2003.

13. Ding S.L., Sheu L.F., Yu J.C., Yang Y.L., Chen B., Leu F.J. and Shen C.Y., Expression of estrogen receptor-alpha and $\mathrm{Ki} 67$ in relation to pathological and molecular features in early-onset infiltrating ductal carcinoma. J. Biomed. Sci. 11: 911-919, 2004.

14. Gold B., Kalush F., Bergeron J., Scott K., Mitra N., Wilson K., Ellis N., Huang H., Chen M., Lippert R., Halldorsson B.V., Woodworth B., White T., Clark A.G., Parl F.F., Broder S., Dean M. and Offit K., Estrogen receptor genotypes and haplotypes associated with breast cancer risk. Cancer Res. 64: 8891-8900, 2004.

15. Shen C.Y., Yu J.C., Lo Y.L., Kuo C.H., Yue C.T., Jou Y.S., Huang C.S., Lung J.C. and Wu C.W., Genome-wide search for loss of heterozygosity using laser capture microdissected tissue of breast carcinoma- an implication for mutator phenotype and breast cancer pathogenesis. Cancer Res. 60: 3884-3892, 2000.

16. Cheng C.W., Wu P.E., Yu J.C., Huang C.S., Yue C.T., Wu C.W. and Shen C.Y., Mechanisms of inactivation of E-cadherin in breast carcinoma: modification of the twohit hypothesis of tumor suppressor gene. Oncogene 20: 3814-3823, 2001.

17. Guilford P., Hopkins J., Harraway J., McLeod M., McLeod N., Harawira P., Taite H., Scoular R., Miller A. and Reeve A.E., E-cadherin germline mutations in familial gastric cancer. Nature 392: 402-405, 1998

18. Kumar R., Wang R.A., Mazumdar A., Talukder A.H., Mandal M., Yang Z., Bagheri-Yarmand R., Sahin A., Hortobagyi G., Adam L., Barnes C.J. and Vadlamudi R.K., A naturally occurring MTAl variant sequesters oestrogen receptor-alpha in the cytoplasm. Nature 418: 654-657, 2002.

19. Yang P.S., Yang T.L., Liu C.L., Wu C.W. and Shen C.Y., A case-control study of breast cancer in Taiwan. Br. J. Cancer 75: 752-756, 1997.

20. Lo Y.L., Yu J.C., Huang C.S., Tseng S.L., Chang T.M., Chang K.J., Wu C.W. and Shen C.Y., Allelic loss of the $B R C A 1$ and $B R C A 2$ genes and other regions on $17 \mathrm{q}$ and $13 \mathrm{q}$ in breast cancer among women from Taiwan (area of low incidence but early onset). Int. J. Cancer 79: 580-587, 1998.

21. Huang C.S., Chern H.D., Chang K.J., Cheng C.W., Hsu S.M. and Shen C.Y., Breast cancer risk associated with genotype polymorphism of the estrogen-metabolizing genes CYP17, CYP1A1, and COMT: a multigenic study on cancer susceptibility. Cancer Res. 59: 4870-4875, 1999.

22. Cheng T.C., Chen S.T., Huang C.S., Fu Y.P., Yu J.C., Cheng C.W., Wu P.E. and Shen C.Y., Breast cancer risk associated with genotype polymorphism of the catechol estrogen-metabolizing genes: a multigenic study on cancer susceptibility. Int. J. Cancer 113: 345-353, 2005.

23. Lung J.C., Chu J.S., Yu J.C., Yue C.T., Lo Y.L., Shen C.Y. and Wu C.W., Aberrant expression of cell cycle regulator cyclin D1 in breast cancer is related to chromosomal genomic instability. Gene Chromosome Cancer 34: 276284, 2002.

24. Fu Y.P., Yu J.C., Cheng T.C., Lou M.A., Hsu G.C., Wu C.Y., Chen S.T., Wu H.S., Wu P.E. and Shen C.Y., Breast cancer risk associated with genotypic polymorphism of the nonhomologous end-joining genes: a multigenic study on cancer susceptibility. Cancer Res. 63: 2440-2446, 2003

25. Lo Y.L., Yu J.C., Chen S.T., Yang H.C., Fann C.S., Mau Y.C. and Shen C.Y., Breast cancer risk associated with genotypic polymorphism of the mitosis-regulating gene Aurora-A/STK15/BTAK. Int. J. Cancer 115: 276-283, 2005.

26. Li L.C., Chui R.M., Sasaki M., Nakajima K., Perinchery G., Au H.C., Nojima D., Carroll P. and Dahiya R., A single nucleotide polymorphism in the E-cadherin gene promoter alters transcriptional activities. Cancer Res. 60: 873-876, 2000.

27. Shin Y., Kim I.J., Kang H.C., Park J.H., Park H.W., Jang S.G., Lee M.R., Jeong S.Y., Chang H.J., Ku J.L. and Park J.G., A functional polymorphism $(-347 \mathrm{G}->\mathrm{GA})$ in the E-cadherin gene is associated with colorectal cancer. Carcinogenesis 25: 2173-2176, 2004. 
28. Ohnishi Y., Tanaka T., Ozaki K., Yamada R., Suzuki H. and Nakamura Y., A high-throughput SNP typing system for genome-wide association studies. J. Hum. Genet. 46: 471-477, 2001.

29. Sellers T.A., Genetic Ancestry and Molecular Epidemiology. Cancer Epidemiol. Biomarkers Prev. 13: 499-500, 2004.

30. Kleinbaum D.G., Kupper L.L. and Morgenstern K.H., Epidemiologic Research: Principles and Quantitative Methods. Van Nostrand Reinhold, NY, 1982 .

31. Kleinbaum D.G., Kupper L.L. and Muller K.E., Applied regression analysis and other multivariable methods. PWSKENT Publishing Company, Boston, 1998.

32. Vineis P., Individual susceptibility to carcinogens. Oncogene 23: 6477-6483, 2004

33. Russo J., Tay L.K. and Russo I.H., Differentiation of the mammary gland and susceptibility to carcinogenesis. Breast Cancer Res. Treat. 2: 5-73, 1982.

34. Johnston S.R. and Dowsett M., Aromatase inhibitors for breast cancer: lessons from the laboratory. Nat. Rev. Cancer 3: 821-831, 2003.

35. Smith I.E. and Dowsett M., Aromatase inhibitors in breast cancer. N. Engl. J. Med. 348: 2431-2442, 2003.

36. Kim M.J., Cardiff R.D., Desai N., Banach-Petrosky W.A., Parsons R., Shen M.M. and Abate-Shen C., Cooperativity of Nkx3.1 and Pten loss of function in a mouse model of prostate carcinogenesis. Proc. Natl. Acad. Sci. USA 99: 2884-2889, 2002.

37. Roberts C.W. and Orkin S.H., The SWI/SNF complexchromatin and cancer. Nat. Rev. Cancer 4: 133-142, 2004.

38. Versteege I., Sevenet N., Lange J., Rousseau-Merck M.F., Ambros P., Handgretinger R., Aurias A. and Delattre O.,
Truncating mutations of hSNF5/INI1 in aggressive paediatric cancer. Nature 394: 203-206, 1998.

39. Gunduz E., Gunduz M., Ouchida M., Nagatsuka H., Beder L., Tsujigiwa H., Fukushima K., Nishizaki K., Shimizu K. and Nagai N., Genetic and epigenetic alterations of BRG1 promote oral cancer development. Int. J. Oncol. 26: 201210, 2005.

40. Medina P.P., Carretero J., Fraga M.F., Esteller M., Sidransky D. and Sanchez-Cespedes M., Genetic and epigenetic screening for gene alterations of the chromatinremodeling factor, SMARCA4/BRG1, in lung tumors. Genes Chromosomes Cancer 41: 170-177, 2004.

41. Bau D.T., Fu Y.P., Chen S.T., Cheng T.C., Yu J.C., Wu P.E. and Shen C.Y., Breast cancer risk and the DNA double-strand break end-joining capacity of non-homologous end-joining genes are affected by BRCA1. Cancer Res. 64: 5013-5019, 2004

42. Hazan R.B., Qiao R., Keren R., Badano I. and Suyama K., Cadherin switch in tumor progression. Ann. NY Acad. Sci. 1014: 155-163, 2004

43. Hatsell S., Rowlands T., Hiremath M. and Cowin P., Betacatenin and Tcfs in mammary development and cancer. J. Mammary Gland Biol. Neoplasia 8: 145-158, 2003.

44. Guilford P., E-cadherin downregulation in cancer: fuel on the fire?. Mol. Med. Today 5: 172-177, 1999.

45. Okubo T., Truong T.K., Yu B., Itoh T., Zhao J., Grube B., Zhou D. and Chen S., Down-regulation of promoter 1.3 activity of the human aromatase gene in breast tissue by zinc-finger protein, snail (SnaH). Cancer Res. 61: 1338-1346, 2001. 